\title{
PERFIL EPIDEMIOLÓGICO DAS NOTIFICAÇÕES DE TUBERCULOSE DE PACIENTES COM RESIDÉNCIA NA 17 REGIONAL DE SAÚDE DO PARANÁ ENTRE 2010 E 2017
}

\author{
Epidemiological profile of notifications of \\ tuberculosis in patients living in the 17th health care \\ region of of the state of Paraná from 2010 to 2017
}

Juliana Zanoni Dotti ${ }^{1}$, Joice Mara Cruciol' ${ }^{2}$, Willian Herbert Noguti de Lima ${ }^{3}$

\begin{abstract}
1. Especialização em Residência em Análises Clínicaspela Universidade Estadual de Londrina, Londrina, Paraná, Brasil (2012) . ORCID: https://orcid.org/0000-0002-0000-4461

2. Doutorado em Medicina e Ciências da Saúde pela Universidade Estadual de Londrina, Londrina, Paraná, Brasil (2006). ORCID: https://orcid.org/0000-0002-2207-8963

3. Administrativo em 17a Regional de Saúde, Londrina, Paraná, Brasil. ORCID: https://orcid.org/0000-0001$8005-5340$
\end{abstract}

CONTATO: Juliana Zanoni Dotti | Alameda Manoel Ribas, nº 01 | Centro | Londrina | Paraná | CEP. 86010-140 | E-mail: judotti@yahoo.com.br

COMO CITAR: Dotti JZ, Cruciol JM, Lima WHN. Perfil epidemiológico das notificações de tuberculose de pacientes com residência na 17ạ Regional de Saúde do Paraná entre 2010 e 2017. R. Saúde Públ. 2018 Dez;1(2):75-82

(c) (i) CopYRIGHT Esta obra é disponibilizada nos termos da Licença Creative Commons - 4. 0

RESUMO Mundialmente a tuberculose mata mais pessoas do que qualquer outra infecção. Obedecer aos esquemas terapêuticos torna a tuberculose curável. Este é um estudo transversal retrospectivo descritivo do perfil epidemiológico dos casos novos de tuberculose notificados na 17ª Regional de Saúde do Paraná no período de 2010 a 2017. A incidência variou entre 28,2 e 35,9 casos/100.000 habitantes. Dos 1.661 casos notificados, predominaram homens brancos com ensino fundamental, 10\% populações especiais (privados de liberdade $7,7 \%$ ). A baciloscopia se apresentou positiva no diagnóstico em 54,6\% casos e 8,8\% não realizaram teste para HIV. Alcoolismo foi o agravo associado mais prevalente (24,2\%). Em 70,1\% dos casos evoluíram para cura e 7,9\% abandonaram o tratamento. Entre data de diagnóstico e a notificação houve diferença média de 10,3 dias em 
51,8\% das notificações. É necessário qualificar a assistência para alcançar a meta de cura de 85\% dos casos proposta pela Organização Mundial da Saúde.

RESUMO Incidência. Tuberculose. Epidemiologia. Notificação de Doenças.

\begin{abstract}
Tuberculosis kills more people than any other infection worldwide. It may be curable if recommended therapeutic regimens are followed. This is a retrospective cross-sectional, descriptive study of the epidemiological profile of new cases of tuberculosis reported in the 17th Healthcare Region of of the state of Paraná from 2010 to 2017 . The incidence varied between 28.2 and 35.9 cases per 100,000 inhabitants. Of the 1,661 new cases reported, white men who finished just primary education were predominant, and $10 \%$ were special populations, with $7.7 \%$ of prisoners. Bacilloscopy was positive in $54.6 \%$ of the cases, and $8.8 \%$ of the sample have not tested for HIV. Alcoholism was the most prevalent associated disease (24.2\%). Of the cases, $70.1 \%$ progressed to cure, and $7.9 \%$ abandoned treatment. Between the date of diagnosis and the notification there was an average difference of 10.3 days in $51.8 \%$ of the reports. It is necessary to qualify the assistance to reach the goal of cure of $85 \%$ of cases proposed by the World Health Organization.
\end{abstract}

KEYWORDS: Incidence. Tuberculosis. Epidemiology. Disease Notification

\title{
INTRODUÇÃO
}

$\mathbf{A}$

tuberculose (TB) é uma doença infecciosa mundialmente preocupante e conhecida desde a antiguidade. ${ }^{1-2} \mathrm{O}$ agente etiológico é o bacilo Mycobacterium tuberculosis, descoberto em 1882 por Robert Koch. Ela afeta tipicamente os pulmões (TB pulmonar), entre outros locais (TB extrapulmonar). ${ }^{2}$

A transmissão da TB é direta, pela tosse, e a aglomeração de pessoas é o principal fator de transmissão. ${ }^{3}$ Subnutrição, tabagismo, alcoolismo, diabetes, infecção pelo vírus da imunodeficiência humana (HIV) favorecem o estabelecimento da TB. ${ }^{4}$

O diagnóstico da TB é baseado em sinais e sintomas clínicos e a confirmação ocorre por baciloscopia e cultura do escarro, além de outros exames auxiliares, como a prova tuberculínica cutânea (PPD) e o exame histológico. ${ }^{2}$

O Esquema Básico (EB) para tratamento da TB consiste em comprimidos de dose fixa combinada (DFC) dos quatro medicamentos: 150 mg de rifampicina (R), 75 mg de isoniazida $(H), 400$ mg de pirazinamida (Z) e 275 mg de etambutol (E) conhecida por fase intensiva (RHZE) com duração de 2 meses. Seguido por uma fase de manutenção $(\mathrm{RH})$ de 4 meses para todos os casos, com exceção dos casos de meningite por TB, onde é utilizado outro esquema. ${ }^{5-6}$ Os casos de falência do tratamento recebem o EB até o resultado dos exames de cultura e teste de sensibilidade aos medicamentos para definição do esquema a ser empregado individualmente, conhecido por Esquema Especial (EE). ${ }^{5-7}$ Os esquemas fazem parte do Programa Nacional de Controle da Tuberculose (PNCT), que aliado à estratégia do Tratamento Diretamente Observado (TDO) são de fundamental importância para a redução da morbidade e mortalidade provocada pela TB. ${ }^{8}$

O objetivo deste estudo foi descrever o perfil epidemiológico presente nas notificações dos casos 
novos de TB de pacientes com residência na $17^{\mathrm{a}}$ Regional de Saúde (RS) do Paraná no período de 2010 a 2017, a fim de contribuir e subsidiar discussões para condutas clínicas mais eficientes diante dos casos suspeitos.

\section{METODOLOGIA}

Estudo transversal retrospectivo descritivo do perfil epidemiológico dos casos novos de TB em pacientes residentes e notificados na $17^{\mathrm{a}} \mathrm{RS}$ do Paraná no período de 01 de janeiro de 2010 a 31 de dezembro de 2017.

O estado do Paraná conta com 22 RS, onde cada uma das regionais tem gerenciamento próprio de um conjunto de municípios. A $17^{\mathrm{a}}$ RS tem sede na cidade de Londrina, atende outros vinte municípios e é a segunda maior regional do estado considerando o número de habitantes. ${ }^{9}$ A TB é uma doença de notificação compulsória no Brasil e a responsabilidade do preenchimento da ficha de notificação é do profissional de saúde.? A fonte dos dados de TB foi o banco de dados do Sistema de Agravos de Notificação (SINAN) ${ }^{10}$, e a de populações foi o Instituto Brasileiro de Geografia e Estatística $\left(\right.$ IBGE) ${ }^{11}$. A ficha de notificação, bem como as definições, são as padronizadas nacionalmente.10 Os registros do banco de dados já se encontravam revisados para duplicidades e outras inconsistências tais como: campos sem preenchimento e erros de digitação.

Foram selecionados os casos novos de TB, de ambos os sexos e maiores de 18 anos. Os critérios de exclusão foram casos de: recidiva, reingresso após abandono do tratamento, transferência do paciente, aqueles notificados após a morte do paciente e os que tinham história prévia desconhecida.

Para caracterização epidemiológica foram avaliadas as seguintes variáveis:

- características sociodemográficas: sexo (masculino e feminino); idade (em anos, categorizada nas faixas etárias: 18 a 29; 30 a 39; 40 a 49; 50 a 59; 60 anos ou mais); raça/cor (branca, preta, amarela, indígena, parda e ignorada); escolaridade (analfabeto, ensino fundamental, médio, superior, e ignorada); populações especiais (privada de liberdade, profissional de saúde, moradores de rua e imigrante);

- características clínicas: data do diagnóstico: forma da TB (pulmonar, extrapulmonar, pulmonar+extrapulmonar); agravos associados (aids, alcoolismo, diabetes melittus, doença mental, uso de drogas ilícitas, tabagismo e outras comorbidades); baciloscopia de escarro (positiva, negativa), cultura do escarro (positivo, negativo), perfil de sensibilidade aos medicamentos do EB (sensivel, resistente).

- características do tratamento: data de início do tratamento; data da notificação; TDO (sim, não); situação no encerramento do caso (cura, abandono, óbito por TB, óbito por outras causas, mudança de diagnóstico, TB-DR, mudança de esquema, falência, abandono primário do tratamento) e data de encerramento.

A análise descritiva dos dados foi feita por meio de frequência para as variáveis qualitativas e dos cálculos de média, desvio padrão, moda e mediana para as variáveis quantitativas. A análise estatística dos dados e gráficos foi realizada no programa Microsoft Office Excel ${ }^{\circledR} 2007$.

A seleção dos casos preservou o sigilo de identificação dos pacientes. 0 estudo foi aprovado pelo Comitê de Ética da Universidade Estadual de Londrina em maio de 2018 com número 84145318.0.0000.5321. Não houve conflito de interesse no estudo e o suporte financeiro foi próprio.

\section{RESULTADOS}

O total de notificações de casos de TB no período de 2010 a 2017 foi de 1.973, e o número de casos novos maiores de 18 anos foi de 1.661. A incidência de TB variou entre 28,2 e 35,9/100 mil habitantes ao ano, a média foi de 32,3 \pm 2,3/100 mil habitantes ao ano (gráfico 1). 
Houve predominância de notificações de pacientes do sexo masculino (1.234; 74,3\%), brancos (1.097; 66\%) e alfabetizados no ensino fundamental (936; 56,4\%). A idade variou entre 18 e 91 anos, com média de 30,5 \pm 14 anos, com maior número de casos na faixa etária de 18 a 29 anos (451; 27,2\%).

As características sociodemográficas estão descritas na tabela 1.

Gráfico 1. Incidência de tuberculose em maiores de 18 anos entre os anos de 2010 e 2017, segundo notificações na $17^{a}$ Regional de Saúde do Paraná.

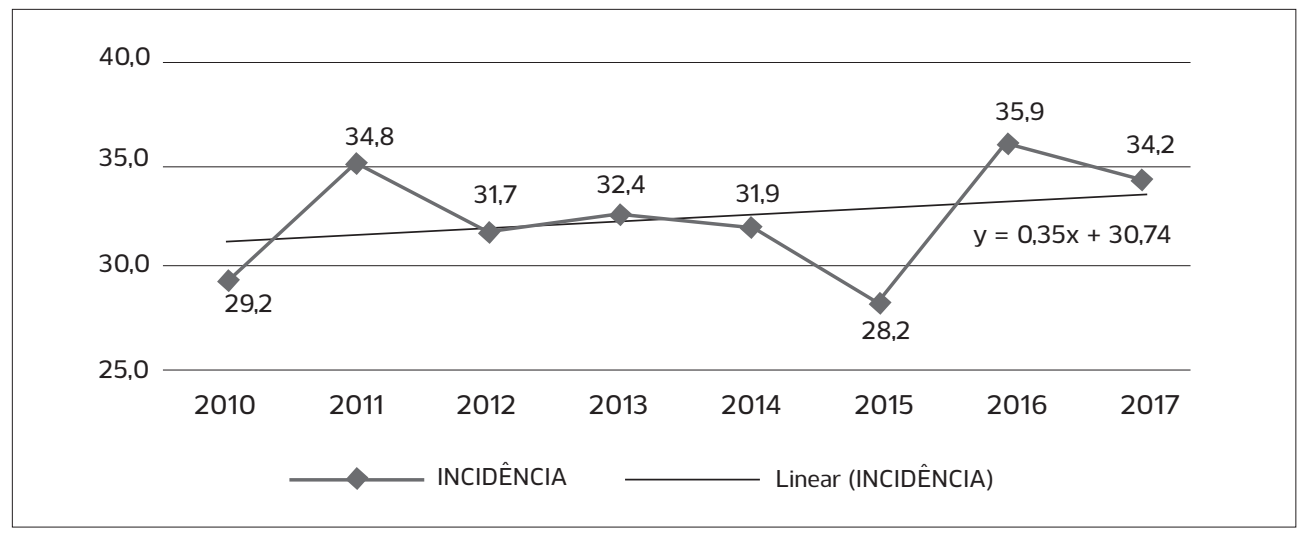

Fonte: Elaborado pelos autores (2018)

Tabela 1. Características sociodemográficas dos casos novos de tuberculose, maiores de 18 anos entre os anos de 2010 e 2017, segundo notificações na $17^{\text {a }}$ Regional de Saúde do Paraná.

\begin{tabular}{ccc}
\hline & $\mathbf{n}$ & $\%$ \\
Sexo & & \\
Feminino & 427 & 25,7 \\
Masculino & 1.234 & 74,3 \\
Faixa Etária & & \\
$18-29$ & 451 & 27,2 \\
$30-39$ & 327 & 19,7 \\
$40-49$ & 334 & 20,1 \\
$50-59$ & 263 & 15,8 \\
$>=60$ & 286 & 17,2 \\
Raça/Cor & & \\
Branca & 1.097 & 66,0 \\
Preta & 151 & 9,1 \\
Amarela & 31 & 1,9 \\
Indígena & 7 & 0,4 \\
Parda & 351 & 21,1 \\
Ignorado & 24 & 1,5 \\
Escolaridade & & \\
Analfabeto & 66 & 4,0 \\
Fundamental & 936 & 56,4 \\
Médio & 312 & 18,8 \\
Superior & 107 & 6,4 \\
Ignorado & 240 & 14,5 \\
\hline
\end{tabular}

Fonte: Elaborado pelos autores (2018)
Da amostra selecionada (1.661 casos), 167 (10\%) faziam parte de populações especiais, onde 31 (1,9\%) moradores de rua, 123 (7,4\%) privados de liberdade, $12(0,7 \%)$ profissionais de saúde e 1 $(0,06 \%)$ imigrante.

Quanto à presença de agravos associados: aids 166 (10\%), uso de drogas ilícitas 139 (8,4\%), tabagismo 250 (15,1\%), alcoolismo 402 (24,2\%), diabetes 141 (8,5\%), doenças mentais 46 (2,8\%). outras comorbidades 325 (19,6\%).

A forma pulmonar foi diagnosticada em 1.304 (78,5\%) casos, a extrapulmonar em 295 (17,8\%) e a combinação delas em 62 (3,7\%) casos. Os tipos e frequência das formas extrapulmonares estão dispostos no gráfico 2 .

No momento do diagnóstico, a baciloscopia do escarro se apresentou: positiva em 907 (54,6\%) casos, negativa em 398 (24\%), não foi realizada em 341 (20,5\%) e não se aplicava em 15 (0,9\%) casos. A cultura no escarro se apresentou: positiva em 441 (26.5\%), negativa em 209 (12,6\%), em andamento em 52 (3,1\%) e não foi realizada em 959 (57,7\%) casos. 0 teste de sensibilidade aos medicamentos 
Gráfico 2 . Frequência das formas extrapulmonares de tuberculose dos casos novos de tuberculose, maiores de 18 anos entre os anos de 2010 e 2017, segundo notificações na 17a Regional de Saúde do Paraná.

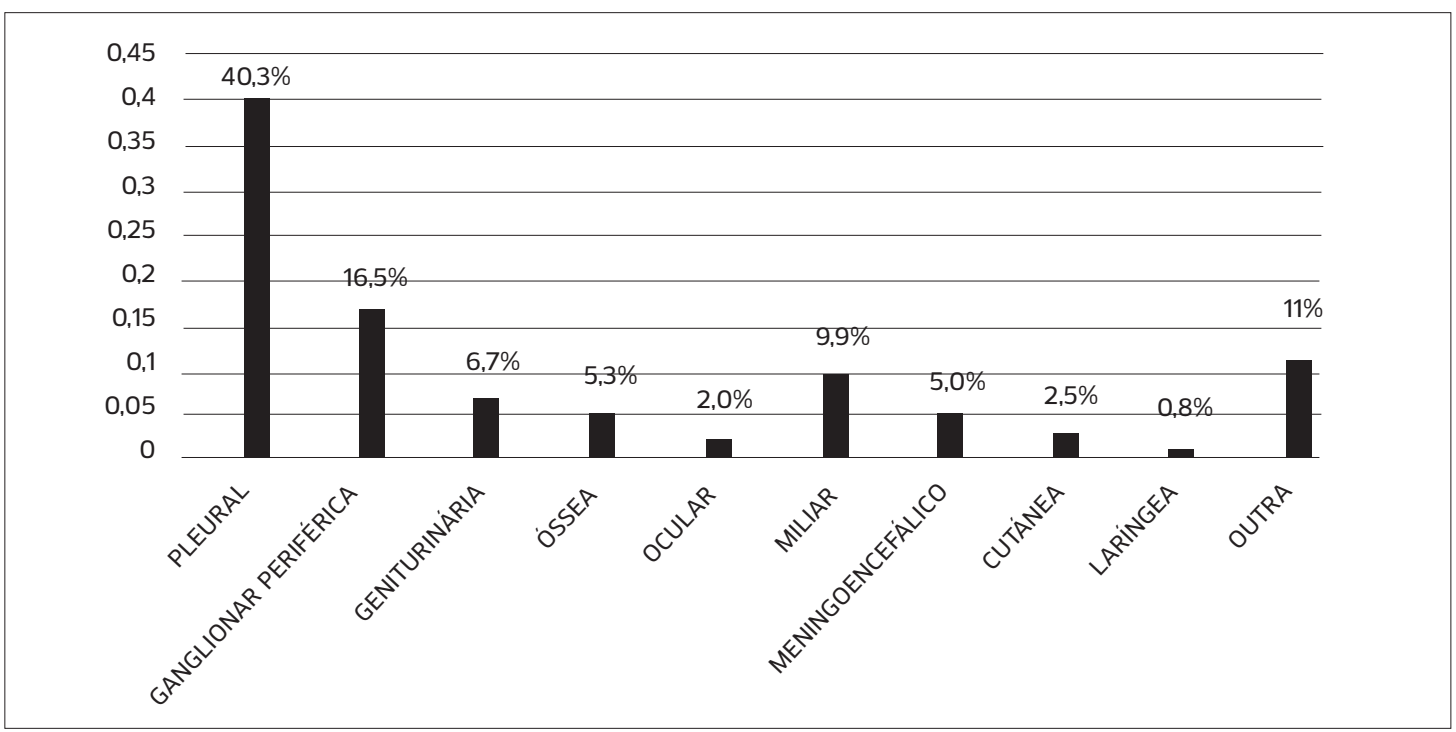

Fonte: Elaborado pelos autores (2018)

Tabela 2. Frequência das situações de encerramento da ficha de notificação dos casos novos de tuberculose, maiores de 18 anos entre os anos de 2010 e 2017, segundo notificações na $17^{\text {a }}$ Regional de Saúde do Paraná.

\begin{tabular}{lcc}
\hline Variável & $\mathbf{n}$ & $\%$ \\
Cura & 1.165 & 70,1 \\
Abandono & 132 & 7,9 \\
Óbito por TB & 55 & 3,3 \\
Óbito por outras causas & 98 & 5,9 \\
Transferência & 69 & 4,2 \\
Mudança de diagnóstico & 52 & 3,1 \\
TB-DR & 40 & 2,4 \\
Mudança de esquema & 14 & 0,8 \\
Falência & 0 & 0 \\
Abandono Primário & 1 & 0,1 \\
Não informado & 35 & 2,1 \\
Total & 1.661 & 100 \\
\hline
\end{tabular}

Fonte: Elaborado pelos autores (2018)

se mostrou resistente em 33 (2\%), sensivel em 229 (13,8\%), estava em andamento em 9 (0,5\%), não foi realizado em 110 (6.6\%) e não foi informado em $1.280(77,1 \%)$ casos.

Das notificações analisadas, em 1.515 (91,2\%) o teste para HIV foi realizado e observaram-se os seguintes resultados: positivo em 177 (11,6\%), negativo em 1.326 (87,5\%) e estava em andamento em $12(0,8 \%)$ casos.

Quanto ao tratamento, 891 (53,6\%) fizeram o TDO, 102 (6,1\%) não fizeram, 1 (0,1\%) caso era ignorado e para $667(40,2)$ casos não havia essa informação. A duração média de tratamento dos casos notificados foi de 6,06 \pm 3,5 meses, moda 6,1 e mediana 6,3. As frequências das situações de encerramento foram dispostas na tabela 2.

Houve uma diferença entre a data do diagnóstico e a data da notificação em 860 (51,8\%) casos, com média de 10,3 $\pm 24,0$ dias. E também uma diferença entre a data do diagnóstico e a data de início do tratamento em 688 (41,4\%) casos, com média de 2,9 $\pm 13,6$ dias.

\section{DISCUSSÃO}

O Brasil está na lista dos 20 países que concentram 90\% dos casos de TB no mundo. ${ }^{2}$ Neste estudo verificou-se que a incidência de TB registrada na $17^{\mathrm{a}} \mathrm{RS}$ do Paraná apresentou aumento de 29,2 para 34,2 casos/100 mil habitantes. No ano de 2015 foi registrada a menor incidência 
do período avaliado. Isso talvez possa ser reflexo da proposta do Brasil para o comitê executivo da Organização Mundial da Saúde (OMS) e que ficou conhecida por Estratégia do Fim da TB, cuja avaliação e aprovação ocorreram entre os anos de 2013 e $2014 .^{12}$

Dados semelhantes de aumento na incidência da TB na $17^{a}$ RS já haviam sido publicados, porém considerando a população geral, independente da idade e indicavam que no ano de 2010 a incidência foi de 25,6 casos e de $32,5 \pm 2,6$ casos $/ 100$ mil habitantes no ano de $20177^{10}$ Já o relatório do Ministério da Saúde (MS) mostrou que no Paraná houve redução da incidência entre 2010 e 2017 que foi de 22,5 casos para 17,2 casos/100 mil habitantes $^{13}$. É possível que tenha ocorrido o deslocamento da população para as regiões mais populosas e aumentado a incidência na $17^{\mathrm{a}} \mathrm{RS}$, mas não temos dados para essa análise e nem para justificar a diminuição da incidência no estado divulgada pelo MS.

Nossos dados mostram predominância em homens brancos com ensino fundamental. Um trabalho similar avaliou 1.418 casos de TB na cidade de Londrina no período de 2001 a 2008 e evidenciou $68,1 \%$ dos casos em homens, 46,5\% na raça branca, 63\% em indivíduos no ensino fundamental, com idade média de 39,8 anos. ${ }^{14}$ Observa-se que a TB acomete mais homens. ${ }^{15}$ As distinções comportamentais e culturais entre homens e mulheres podem estar relacionadas a esses dados. ${ }^{16}$ A faixa etária mais prevalente do estudo foi de adultos jovens, estando de acordo com estudos epidemiológicos publicados no Brasil e na África do Sul, países do grupo BRICS (Brasil, Rússia, Índia, China e África do Sul). ${ }^{15}$

No quesito populações especiais, foram encontradas proporções muito baixas de preenchimento da informação. Mais da metade (884; 53,3\%) dos registros das variáveis não foi preenchida e apenas 10\% (167) dos pacientes constavam como pertencentes a alguma população especial, com prevalência dos privados de liberdade. Estudo semelhante desenvolvido no período de 2001 a 2014 também evidenciou que 93 a 99,4\% dos registros dessas variáveis encontravam-se como dado ignorado ou não preenchido. ${ }^{17}$ Estudo recente demonstrou que dos detentos avaliados, 14\% foram diagnosticados com TB ativa. ${ }^{18}$ Estar preso por período maior que seis meses é um fator que se relaciona ao adoecimento por TB, e o doente de TB pode vir a transmitir a doença a outros detentos, aos profissionais que trabalham no sistema prisional, aos visitantes e a indivíduos na comunidade.19-20

A TB é geralmente resultado da debilidade do siste ma imunológico do paciente causada por doenças, comorbidades e hábitos. ${ }^{21}$ Foi observado que dentre as doenças e agravos associados à TB, alcoolismo 24,2\% (402) e tabagismo 15,1\% (250) foram predominantes, considerando que essa informação não estava disponível em 53,4\% (887) das notificações para a variável tabagismo. Estudo realizado em Taiwan encontrou aumento de duas vezes no risco de TB ativa em fumantes comparados a não fumantes, e $17 \%$ dos casos de TB naquela população estudada eram atribuídos ao tabagismo. ${ }^{22}$ E um estudo realizado em Carapicuíba, São Paulo, constatou o alcoolismo como comorbidade de maior prevalência nos casos de TB e a associou como fator de risco mais significativo para o mau prognóstico e desfecho da doença. ${ }^{19}$

Segundo dados da OMS, aproximadamente 85\% dos casos de TB são de localização pulmonar e, devido aos sintomas clínicos apresentados serem gerais, os pacientes costumam buscar serviços de atenção primária. ${ }^{12}$

Dados do MS sobre o estado do Paraná mostrou que em 2017, a testagem para HIV entre os casos novos de TB foi de $84,4 \% .^{13}$ No presente trabalho, 91,2\% (1.515) foram testados para HIV, valor próximo aos números do estado. A oferta do teste para HIV, quando feita de forma adequada, pode representar mudança em termos de redução de mortalidade por coinfecção HIV/TB, pois contribui para o planejamento de assistência ao portador $^{23}$. Vale ressaltar que um terço das mortes relacionadas ao HIV são decorrentes de TB. ${ }^{8}$

Mais da metade dos casos receberam os medicamentos por TDO e tiveram percentual de cura de 74\% (660/891) e de abandono 9,8\% (88/891). Para os que não receberam TDO 
(6\%; 102/1.661), o encerramento do caso foi de cura para 75,5\% (77/102) e de abandono para 10,8\% (11/102) dos casos. A estratégia TDO se fundamenta na observação direta da tomada do medicamento, três vezes por semana nos dois primeiros meses, seguido por uma vez por semana até o final do tratamento. Apesar de favorecer a adesão ao tratamento, revisões sistemáticas não conseguiram demonstrar maior efetividade do TDO em comparação ao tratamento auto-administrado, provavelmente porque a efetividade do tratamento é dependente de vários fatores e não apenas da tomada do medicamento. ${ }^{24}$ Essa afirmação condiz com os dados encontrados nesse estudo. Todavia, nos Estados Unidos e Europa o TDO é recomendado por estar associado à negativação da baciloscopia durante o tratamento. ${ }^{25}$

O tratamento da TB é complexo, com duração média de seis meses, e deve ser mantido mesmo após a remissão dos sinais e sintomas, que ocorre em média inferior a sessenta dias pósinício do tratamento. Em razão disso, após esse período, verifica-se uma diminuição na adesão ao tratamento. ${ }^{15}$ Neste estudo, a duração média foi condizente com o preconizado pelo MS, contudo não foi investigada a associação entre abandono e o TDO.

Considerando as metas de cura (>85\%) e abandono (<5\%) preconizadas pela $\mathrm{OMS}^{6}$, tanto a $17^{\mathrm{a}} \mathrm{RS}$, quanto o estado do Paraná e o Brasil como um todo, têm apresentado taxas de cura abaixo da meta (57,2\%, 75,8\% e 73\%, respectivamente). Já as taxas de abandono da $17^{\mathrm{a}} \mathrm{RS}$ e do estado do Paraná têm se mostrado próximas da meta e bem distantes dos dados do Brasil como um todo (5,5\%; $5,7 \%$ e $10,3 \%$ respectivamente). ${ }^{13}$

Uma avaliação do Programa de Controle da Tuberculose (PCT) em Divinópolis, Minas Gerais, observou falhas na distribuição de medicamentos e foi sugerido que a descentralização da medicação para as unidades de atenção primária à saúde pode melhorar o desempenho do programa. ${ }^{23}$ No presente estudo detectamos uma pequena diferença entre a data do diagnóstico e a data do início do tratamento, podendo aumentar a eficiência do tratamento. Aquela mesma avaliação referenciou deficiências em municípios brasileiros nas ações de vigilância epidemiológica e na inadequação do processo de notificação. ${ }^{23} \mathrm{Na} 17^{\mathrm{a}}$ RS em mais de 50\% dos casos houve diferença média maior que uma semana entre a data de diagnóstico e data da notificação, evidenciando a necessidade de treinamento das equipes em saúde sobre a importância da notificação e início do tratamento o quanto antes.

\section{CONSIDERAÇÕES FINAIS}

A maior limitação do estudo é a falta de dados completos, decorrente do não preenchimento dos campos presentes na ficha de notificação. Essa inconsistência pode ter subestimado as prevalências reais das variáveis.

Ao descrever o perfil epidemiológico presente nas notificações de TB da $17^{\mathrm{a}} \mathrm{RS}$, evidenciou-se que a taxa de cura piorou significativamente, e em contrapartida a taxa de abandono melhorou muito ao longo dos anos estudados. Em vista disso, a qualidade da assistência prestada pelos profissionais da área necessita de contínuo aperfeiçoamento a fim de atingirmos melhor qualidade de saúde dos habitantes.

\section{REFERÊNCIAS}

1 Maciel MS, Mendes PD, Gomes AP. Siqueira-Batista R. A história da tuberculose no Brasil: os muitos tons (de cinza) da miséria. Rev Bras Clin Méd [Internet]. 2012 [citado 2018 Jun 13]; 10(3): 22630. Disponivel em: http://files.bvs.br/upload/S/1679-1010/2012/ v10n3/a2886.pdf

2 Organização Mundial da Saúde. Relatório Global da Tuberculose de 2017. Geneva: OMS, 2017.

3 Ministério da Saúde (BR), Secretaria de Vigilância em Saúde Departamento de Vigilância das Doenças Transmissiveis. Brasil livre da Tuberculose: Plano Nacional para o Fim da Tuberculose como Problema de Saúde Pública. Brasília: Ministério da Saúde, 2017.

4 Shete PB, Haguma P, Miller CR, Ochom E, Ayakaka I, Davis JL, et al. Pathways and costs of care for patients with tuberculosis symptoms in rural Uganda. Int J Lung Dis [Internet]. 2015 [citado 2018 Ago 13]; 19(8): 912-7. Disponivel em: http://www.ingentaconnect.com/ content/iuatld/ijtld/2015/00000019/00000008/ara00009:jses sionid=130rbko68ktcr.x-ic-live-02. 
5 Ministério da Saúde (BR), Secretaria de Vigilância em Saúde, Programa Nacional de Controle da Tuberculose. Manual de recomendações para o controle da tuberculose no Brasil. Brasília: Ministério da Saúde, 2011

6 Ministério da Saúde (BR), Secretaria de Vigilância em Saúde. Guia de Vigilância em Saúde. Brasilia: Ministério da Saúde, 2014.

7 Rabahi MF, Junior JLRS. Ferreira ACG. Tannus-Silva DGS, Conde MB. Tratamento da tuberculose. J Bras Pneumol [Internet]. 2017 [citado 2018 Ago 15]; 43(5):472-86. Disponivel em: http://dx.doi. org/10.1590/S1806-37562016000000388

8 Barreira D. Os desafios para a eliminação da tuberculose no Brasil. Epidemiol Serv Saúde [Internet]. 2018 [citado 2018 Ago 19]; 27(1). Disponível em: http://dx.doi:10.5123/S167949742018000100009

9 Secretaria de Estado da Saúde do Paraná (PR). Plano Estadual de Saúde do Paraná 2016-2019. Paraná: Secretaria de Estado da Saúde, 2016.

10 Ministério da Saúde (BR). Sistema de informação de agravos de notificação. Brasília: Ministério da Saúde, 2018.

11 Ministério do Planejamento, Orçamento e Gestão (BR). Instituto Brasileiro de Geografia e Estatística. Brasília: Ministério do Planejamento, Orçamento e Gestão, 2010.

12 Organização Mundial da Saúde. Relatório Global da Tuberculose de 2013: Grupo consultivo estratégico técnico para a tuberculose. Geneva: OMS, 2013.

13 Ministério da Saúde (BR), Secretaria de Vigilância em Saúde. Implantação do Plano Nacional pelo Fim da Tuberculose como Problema de Saúde Pública no Brasil: primeiros passos rumo ao alcance das metas. Bolet. Epid. 2018 Mar;49(11):1-18

14 Costa WL Jr. Avaliação dos casos de tuberculose notificados no município de Londrina no período de 2001 a 2008 [dissertação] Londrina: Universidade Estadual de Londrina - UEL; 2011. 51 p. Disponivel em: http://www.uel.br/pos/mpsaude/diss/diss/20.pdf

15 Setolim MA, Andreoni S, Vieira AA. Aspectos epidemiológicos e adesão ao tratamento de tuberculose segundo coinfecção do HIV em pacientes do programa de controle da tuberculose de município prioritário do estado de São Paulo. Rev Bras Pesq Saúde [Internet]. 2015 [citado 2018 Ago 18]; 17(1): 38-47. Disponível em: https://doi.org/10.21722/rbps.v17i1.12448

16 Lemos LA, Feijão AR, Gir E, Galvão MTG. Aspectos da qualidade de vida

de pacientes com coinfecção HIV/tuberculose. Acta Paul Enferm [Internet]. 2012 [citado 2018 Ago 18]; 25(1):41-7. Disponível em: http://dx.doi.org/10.1590/S0103-21002012000800007.

17 Soares MLM, Amaral NAC do, Zacarias ACP, Ribeiro LKNP. Aspectos sociodemográficos e clínico-epidemiológicos de abandono do tratamento de tuberculose em Pernambuco, Brasil, 2001-2014. Epidemiol Serv Saúde [Internet]. 2017 [citado 2018 Ago 12]; 26(2): 369-78. Disponivel em: http://dx.doi.org/10.5123/ s1679-49742017000200014

18 Rocha JZ da, Valença MS, Carrion LL, Silva LV da, Von Groll A. Silva PA. Respiratory symptoms and active tuberculosis in a prison in Southern Brazil: Associated epidemiologic variables. Rev Epidemiol Control Infect [Internet]. 2013 [citado 2018 Ago 16]; 3(4):128-33. Disponivel em: https://online.unisc.br/seer/index.php/ epidemiologia/article/viewFile/3963/3256

19 Vieira AA, Ribeiro SA, Siqueira AM de, Galesi VMN, Santos LAR dos, Golub JE. Prevalence of patients with respiratory symptoms through active case finding and diagnosis of pulmonary tuberculosis among prisoners and related predictors in a jail in the city of Carapicuiba, Brazil. Rev Bras Epidemiol [Internet]. 2010 [citado 2018 Ago 12]:13(4):641-50. Disponivel em: http://dx.doi. org/10.1590/S1415-790X2010000400009.

20 Nogueira PA, Abrahão RMCM. A infecção tuberculosa e o tempo de prisão da população carcerária dos Distritos Policiais da zona oeste da cidade de São Paulo. Rev Bras Epidemiol [Internet]. 2009[citado 2018 Ago 12]: 12(1): 30-8. Disponivel em: http:// dx.doi.org/10.1590/S1415-790X2009000100004

21 Arbex MA, Varella MCL. Siqueira HR de, Mello FAF de. Drogas antituberculose: interações medicamentosas, efeitos adversos e utilização em situações especiais. Parte 1: fármacos de primeira linha. J Bras Pneumol [Internet]. 2010 [citado 2018 Ago 19]; 36(5): 626-40. Disponivel em: http://www.scielo.br/pdf/jbpneu/v36n5/ en_v36n5a16.pdf

22 Chi-Pang W, Ta-Chien C, Hui-Ting C, Min-Kuang T, Ting-Yuang C. Shan-Pou T. The reduction of tuberculosis risks by smoking cessation. BMC Infect Dis [Internet]. 2010 [citado 2018 Ago 13]:(10):156-65. Disponível em: https://doi.org/10.1186/1471-2334$10-156$

23 Andrade HS, Oliveira VC, Gontijo TL, Pessôa MTC, Guimarães EAA. Avaliação do Programa de Controle de Qualidade da Tuberculose: um estudo de caso. Saúde Debate [Internet]. 2017 [citado 2018 Ago 17]: (41):242-58. Disponível em: http://dx.doi. org/10.1590/0103-11042017s18

24 Karumbi J, Garner P. Directly observedt herapy for treating tuberculosis. The Cochrane Database Syst Rev [Internet]. 2015 [citado 2018 Ago 19];(5). Disponivel em: https://doi. org/10.1002/14651858.CD003343.pub4

25 Seaworth BJ, Armitige LY, Griffith DE. First do no harm-adverse events, drug intolerance, and hepatotoxicity: how can we not justify directly observed therapy for treating tuberculosis? Clin Infect Dis [Internet]. 2013[citado 2018 Ago 20]; 57( 7):106364. Disponivel em: https://doi.org/10.1093/cid/cit432. 